\title{
UTILIZAÇÃO DA FOTOCATÁLISE NA ETAPA DE ESTERIFICAÇÃO DE ÁCIDOS GRAXOS PARA A PRODUÇÃO DE BIODIESEL
}

\author{
M. C. MANIQUE ${ }^{1}$, A. P. SILVA ${ }^{1}$, A. K. ALVES ${ }^{1}$, C. P. BERGMANN ${ }^{1}$ \\ ${ }^{1}$ Universidade Federal do Rio Grande do Sul, Escola de Engenharia, Laboratório de Materiais \\ Cerâmicos \\ E-mail para contato: marciamanique@yahoo.com.br
}

\begin{abstract}
RESUMO - A etapa de esterificação pode ser utilizada para matérias-primas de baixo valor agregado, como óleos e gorduras residuais, que apresentam alto teor de ácidos graxos. Neste trabalho, a fotocatálise foi a técnica utilizada para os ensaios de esterificação a partir do ácido oleico. Avaliaram-se as condições de reação, como concentração do catalisador, do ácido oleico e do metanol, utilizando o catalisador comercial P25 (nanopartículas de $\mathrm{TiO}_{2}$ ). A melhor conversão em éster foi obtida pelo catalisador P25 na concentração de $15 \%(\mathrm{~m} / \mathrm{m})$ e razão molar 3:1 (metanol: ácido oleico), chegando ao valor de $86 \%$ em oleato de metila. Além disso, é proposto um mecanismo de reação para o processo de esterificação por fotocatálise.
\end{abstract}

\section{INTRODUÇÃO}

A tendência mundial sobre a redução da dependência dos combustíveis fósseis e a crescente preocupação ambiental tem motivado as pesquisas em biodiesel para a substituição do petróleo diesel (Alenezi et al., 2013; Marchetti e Errazu, 2008; Yan et al., 2009). O biodiesel apresenta características importantes como não-toxicidade, biodegradabilidade, alto teor energético e ausência de enxofre (Corro et al., 2010; Canakci e Van Gerpen, 2001).

O principal processo para a produção de biodiesel é o da transesterificação ou alcoólise, no qual o triglicerídeo reage com um álcool na presença de um catalisador formando ésteres alquílicos (biodiesel) e glicerina. O processo mais utilizado pela indústria é o da transesterificação em meio alcalino devido ao rendimento, tempo de reação e viabilidade econômica. O catalisador e o álcool mais empregados nesta rota são o hidróxido de potássio $(\mathrm{KOH})$ e o metanol, respectivamente (Meher et al., 2006).

Óleo de fritura é um resíduo indesejável, mas que pode ser usado como matéria-prima para a produção de biodiesel, permitindo, assim, a geração de uma fonte alternativa de energia e a redução no impacto ambiental (Canakci e Van Gerpen, 2001). No entanto, o óleo residual apresenta um alto índice de ácidos graxos livres devido à reação de hidrólise dos triglicerídeos com água. O processo de catálise alcalina é muito sensível ao elevado teor de ácidos graxos 


\section{9 a 22 de outubro de 2014 \\ Florianópolis/SC}

livres. Sua presença leva à formação de sabão e ao menor rendimento em biodiesel (Aranda et al., 2008).

Para evitar a reação de saponificação, uma etapa de esterificação é necessária para a conversão dos ácidos graxos em ésteres. Assim, o método mais comum é a utilização de catalisadores ácidos, como ácido sulfúrico $\left(\mathrm{H}_{2} \mathrm{SO}_{4}\right)$, para a reação de esterificação. Este processo é conhecido como um pré-tratamento do óleo. O produto esterificado (no qual deve possuir um índice de acidez inferior a $2 \%$ ) é utilizado para a reação de transesterificação alcalina. O processo em duas etapas permite a produção de biodiesel a partir de óleos com alto teor de ácidos graxos livres. No entanto, a utilização de um catalisador ácido exige uma quantidade muito maior de metanol em comparação com a rota alcalina, necessita de um tempo maior por se tratar de uma reação mais lenta, gera resíduos aquosos de difícil tratamento e ocasiona um alto custo em equipamentos devido à corrosão (Canakci e Van Gerpen, 2001; Corro et al., 2011).

Um processo fotocatalítico heterogêneo pode também ser realizado para obter-se uma eficiente esterificação de ácidos graxos, com a vantagem de não gerar efluentes (Marchetti e Errazu, 2008; Yan et al., 2009). Corro et al. (2013) utilizaram o fotocatalisador $\mathrm{ZnO} / \mathrm{SiO}_{2}$ para a esterificação de ácidos graxos livres presentes no óleo bruto de Jatropha Curcas. O fotocatalisador foi considerado altamente ativo para o processo de esterificação, com a vantagem de que ele pode ser reutilizável, uma vez que a atividade catalítica se mantém praticamente inalterado, mesmo depois de 10 ciclos de utilização. Obteve-se uma conversão de $96 \%$ em ésteres com 4 horas de reação, utilizando uma proporção em massa de catalisador:óleo de $15 \%$ e razão molar metanol:óleo de 12:1.

Com base nestas considerações, os objetivos deste estudo foram: (i) investigar o uso de $\mathrm{TiO}_{2}$ no processo de fotocatálise para esterificação de ácidos graxos; (ii) avaliar as condições de reação de esterificação, como concentração do catalisador, do ácido oleico e do metanol; e (iii) propor um mecanismo de reação para o processo de esterificação fotocatalítica.

\section{MATERIAIS E MÉTODOS}

\subsection{Materiais}

Os reagentes metanol (Synth) e ácido oléico (Vertec) foram de grau analítico. Os padrões cromatográficos foram adquiridos da Sigma-Aldrich. $O$ gás utilizado nas análises cromatográficas (He, com pureza superior a 99\%) foi adquirido da White Martins. Como fotocatalisador padrão, nanopartículas de $\mathrm{TiO}_{2}$ (P25) (80\% anatase e rutilo 20\%) foram adquiridos da Evonik.

\subsection{Esterificação do ácido oleico por fotocatálise}

Para os testes de esterificação e melhor compreensão nos estudos e mecanismos de reação foi utilizado o ácido oleico como reagente. Tal ácido graxo foi escolhido devido a sua presença 
comum nos principais óleos vegetais utilizados para produção de biodiesel. A reação de esterificação do ácido oleico com metanol, na presença do catalisador $\mathrm{TiO}_{2} \mathrm{P} 25$, foi realizado através do processo de fotocatálise, formando, no final da reação, oleato de metila e água.

Os experimentos foram realizados em um sistema fotocatalítico, apresentado pela Figura 1, que consiste em um reator de vidro pyrex encamisado, dois suportes para irradiação de luz UV (em formato de meio-cilindros) possuindo no total 12 lâmpadas negras (Sylvania) de 8W e uma placa de agitação magnética. O reator de vidro possui um orifício vedado por um septo de silicone para a retirada de amostras. A temperatura do reator é mantida constante $\left(30^{\circ} \mathrm{C}\right)$ através de uma entrada e saída de água alimentada por um circulador de água termostatizado (Thermo Scientific).

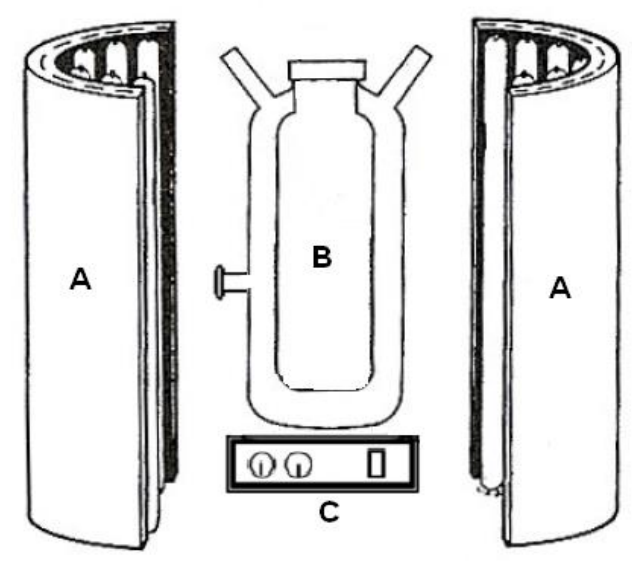

Figura 1 - Representação do sistema fotocatalítico empregado para os ensaios de esterificação.

(A) suportes das lâmpadas UV, (B) reator de vidro e (C) agitador magnético.

Estequiometricamente, a razão molar entre o metanol e o ácido oleico é de 1:1. No entanto, a reação de esterificação é reversível. Para assegurar que a reação ocorra no sentido dos ésteres metílicos, usa-se um excesso estequiométrico de metanol na reação. Desta forma, de acordo com a razão molar metanol:ácido oleico, foram preparadas soluções 3:1, 6:1, 9:1 e 12:1. As concentrações de catalisador estudadas foram de 5, 10, 15 e 20\% (m/m ácido oleico), seguindo o método de Corro et al. (2013). Cada solução foi transferida para o reator fotocatalítico, onde foi mantido sob irradiação UV e agitação constante, a temperatura de $30^{\circ} \mathrm{C}$. O tempo de reação foi de 4 horas. Uma amostra em branco foi preparada com ausência de luz UV e de catalisador. Outra amostra em branco foi preparada sem a presença de luz UV, mas com presença de catalisador na concentração de $15 \%$. Uma amostra foi preparada sem o catalisador $(0 \%)$ com as mesmas condições experimentais.

Terminada a reação, as amostras foram transferidas para tubos de $15 \mathrm{~mL}$ e centrifugadas por 30 minutos (4000 rpm) em uma centrífuga (Quimis) para separar o catalisador da solução. Após a separação, as amostras foram aquecidas a $110^{\circ} \mathrm{C}$ por 15 minutos para remover a água 
resultante da reação de esterificação e também o excesso de metanol utilizado na solução. As amostras foram então armazenadas em frascos âmbar para posteriores análises.

\subsection{Caracterização da conversão em oleato de metila}

O cromatógrafo gasoso Shimadzu, modelo GC-17A, acoplado ao detector de espectrometria de massa Shimadzu, modelo 5050A foi utilizado para determinação da presença do oleato de metila em sua composição.

Para a determinação do teor de éster, foi preparada uma curva de calibração através da injeção de soluções com concentrações conhecidas. A curva de calibração gerada relaciona a área dos picos do oleato de metila com a concentração da solução injetada.

\section{RESULTADOS E DISCUSSÃO}

\subsection{Caracterização do oleato de metila}

Os resultados dos ensaios de esterificação do ácido oleico por fotocatálise de acordo com o teor de catalisador são apresentados pela Figura 2 .

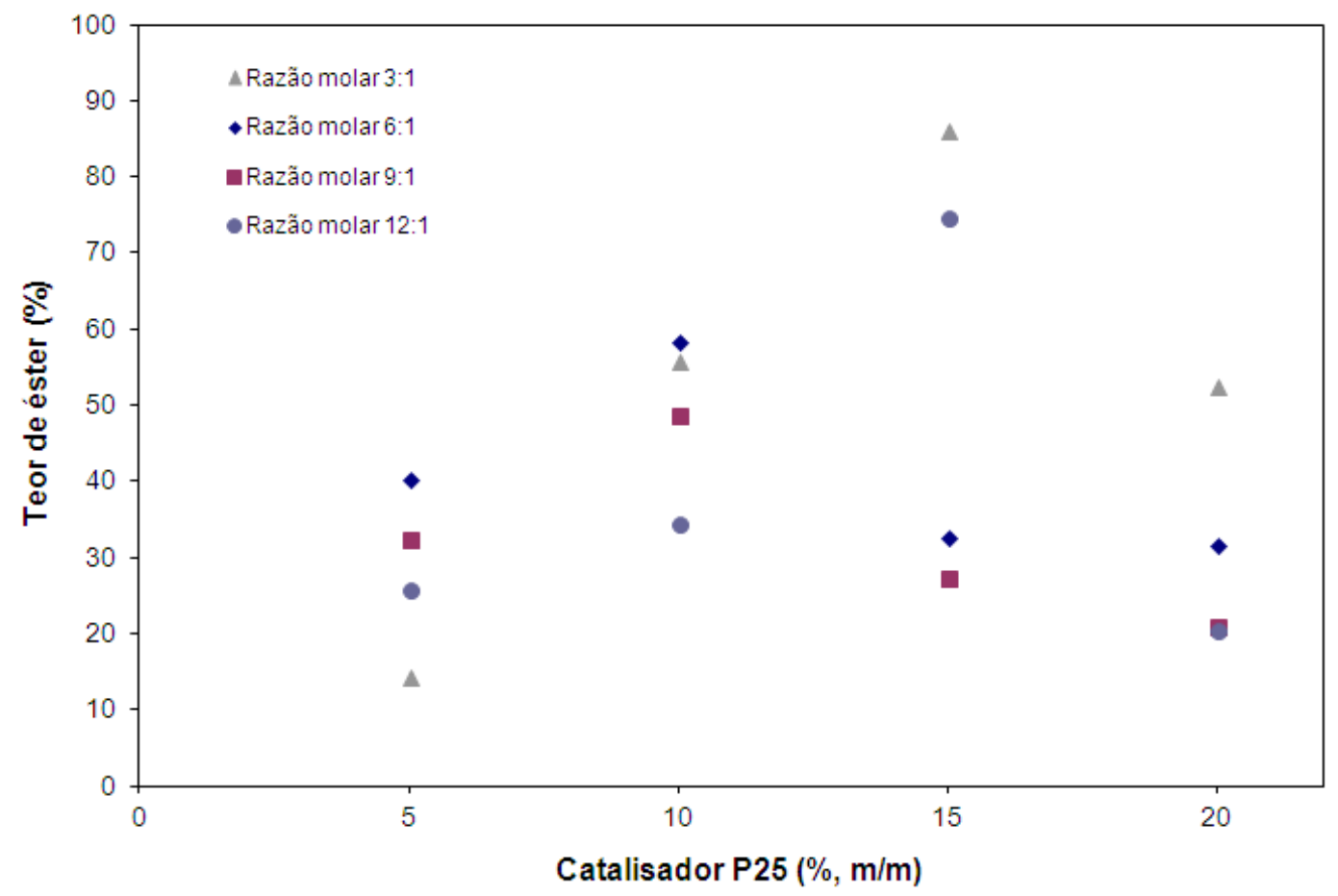

Figura 2 - Resultados do teor de oleato de metila após a reação por fotocatálise, utilizando o catalisador P25. 
De acordo com os resultados apresentados no gráfico, a melhor conversão em éster (oleato de metila) foi a amostra com razão molar de 3:1 (metanol:ácido oleico) e 15\% de catalisador, com uma esterificação de 86,0\%. Assim, não é necessário utilizar um grande excesso estequiométrico de metanol para ocorrer a reação de esterificação.

As amostras 6:1 e 9:1 15\% apresentaram uma diminuição na eficiência de esterificação. Já para a amostra 12:1, houve novamente um aumento. Isto pode ser explicado pela facilidade de interação na emulsão do catalisador com os reagentes e também pela maior incidência de luz UV devido a maior diluição do metanol. De acordo com Corro et al. (2013), para a esterificação ocorrer com altos índices de conversão, é necessário uma concentração de catalisador maior que $10 \%$ em massa, empregando $\mathrm{ZnO} / \mathrm{SiO}_{2}$. Não há ainda estudos sobre a utilização do $\mathrm{TiO}_{2}$ para o processo de esterificação.

Há uma queda na conversão em éster em todas as amostras onde foram utilizados $20 \%$ de catalisador. A concentração de catalisador pode ter elevado saturação da solução, prejudicando essencialmente a absorção de luz UV pelo fotocatalisador e, consequentemente, impedindo sua reação com o ácido oleico e metanol. De acordo com Gogate e Pandit (2004), o aumento da concentração de catalisador gera um crescimento de partículas irradiadas até que se alcance uma concentração crítica, onde todas as partículas estão iluminadas. A partir deste ponto, uma maior concentração de catalisador aumenta a opacidade do sistema reacional, provocando a diminuição da penetração da luz na solução, levando a uma queda na eficiência do processo.

A amostra em branco preparada em ausência de luz UV e catalisador obteve uma conversão de $3,4 \%$ em éster. A amostra preparada sem a presença de luz UV e com $15 \%$ de catalisador obteve o valor de $10,6 \%$.

A amostra preparada sem a presença de catalisador $(0 \%)$ e com presença de luz UV apresentou uma esterificação de 32,6\%. Uma diferença significativa para a amostra onde não houve a presença de luz UV (3,4\%). Isto significa que ocorre a reação de esterificação sem a presença do catalisador, mas ainda com um rendimento menor em presença deste para algumas amostras. A reação que ocorre neste caso é a reação de fotólise, ou seja, a reação ocorre apenas pelo efeito da radiação UV. Hwu et al. (2004) utilizaram o mecanismo da fotólise para a esterificação de ácidos carboxílicos, utilizando vários álcoois. Em seu estudo, os melhores resultados necessitaram em um grande excesso de álcool (razão molar de 33:1 a 400:1), obtendo rendimentos de 66 a $99 \%$.

\subsection{Mecanismo de reação do $\mathrm{TiO}_{2}$ na esterificação do ácido oleico}

A reação de esterificação, onde o ácido graxo reage com um álcool formando éster e água, é o mecanismo geral da esterificação fotocatalítica. No entanto, um estudo minucioso das etapas da reação da fotocatálise no processo de esterificação pode ajudar a aperfeiçoar a produção dos ésteres. 
Primeiramente, sabe-se que após a captura de luz UV, o $\mathrm{TiO}_{2}$ (semicondutor) é excitado por fótons (hv) com energia igual ou superior ao nível de energia da banda. Os elétrons ( $\mathrm{e}^{-}$) recebem a energia do fóton e são promovidos da valência para a banda de condução (BC), gerando lacunas positivas $\left(\mathrm{h}^{+}\right)$na banda de valência (BV). Assim, o par elétron-lacuna difunde para a superfície do semicondutor, tornando-o capaz de reações de transferência de elétrons, uma vez que eles interagem com as moléculas da solução. Se essa interação não ocorre, os pares se recombinam, dissipando como calor.

Os álcoois são muitas vezes adicionados na reação de fotocatálise como agentes de sacrifício para a produção de $\mathrm{H}_{2}$, reduzindo a recombinação das lacunas positivas com os elétrons. Isto permite que os elétrons estejam disponíveis na superfície do catalisador, favorecendo a redução da água. A foto-oxidação do metanol utilizando $\mathrm{TiO}_{2}$ é uma das reações mais estudadas da fotocatálise. Em alguns trabalhos, como de Cui et al. (2005) e Wu et al. (2008), foi proposto um mecanismo da oxidação do metanol, no qual ocorre a formação de radicais $\mathrm{CH}_{3} \mathrm{O} \cdot$ pela captura de lacunas aprisionadas em sítios $\mathrm{Ti}^{4+}-\mathrm{O}$.

A seguir, uma proposta de mecanismo da reação:

1) Na banda de valência, o metanol reage com a lacuna positiva através de sua molécula de oxigênio, formando uma molécula altamente reativa de metóxi e um próton. A reação desta etapa de oxidação é mostrada pela Equação 1:

$$
\mathrm{CH}_{3} \mathrm{OH}+h^{+} \longrightarrow \mathrm{CH}_{3} \mathrm{O}+\mathrm{H}^{+}
$$

2) $\mathrm{O}$ ácido graxo reage com o elétron livre na banda de condução, gerando o radical $\mathrm{RCOOH}^{*}$. Este mecanismo foi mencionado por Corro et al. (2013) conforme a Equação 2:

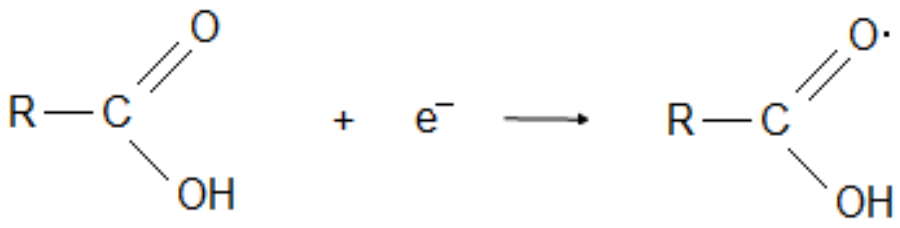

3) Os radicais $\mathrm{RCOOH} \cdot$ atacam os prótons gerados e os radicais $\mathrm{CH}_{3} \mathrm{O} \cdot$ atacam o carbono polarizado, conforme a Equação 3: 


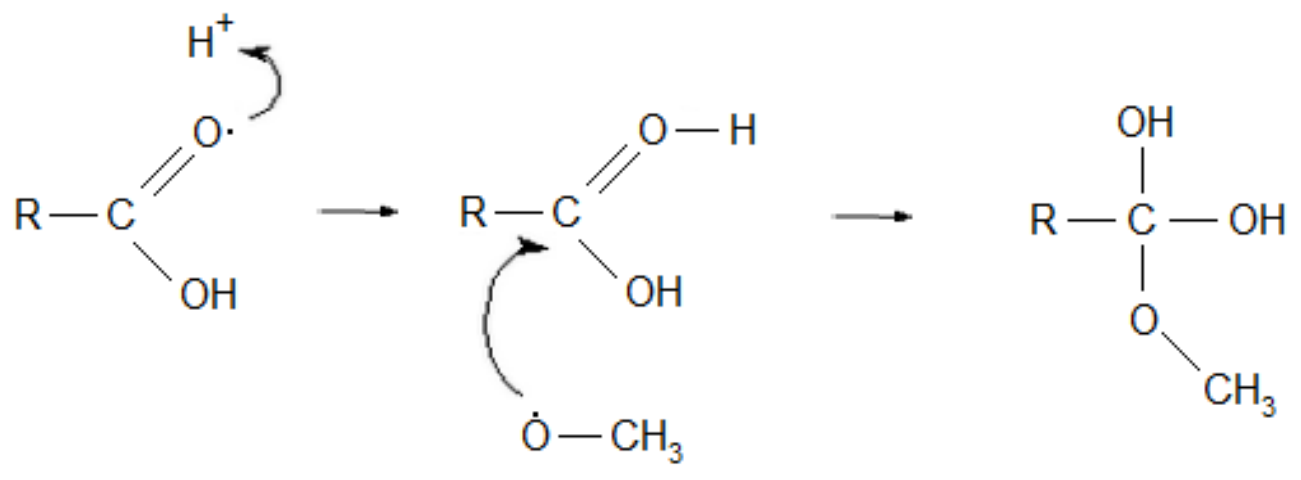

4) O grupo hidroxila ligado ao átomo de carbono pode ligar-se ao átomo de hidrogênio ligado ao oxigênio para formar uma molécula de água. $\mathrm{O}$ rearranjo faz produzir a estrutura molecular do éster, como mostra a Equação 4:

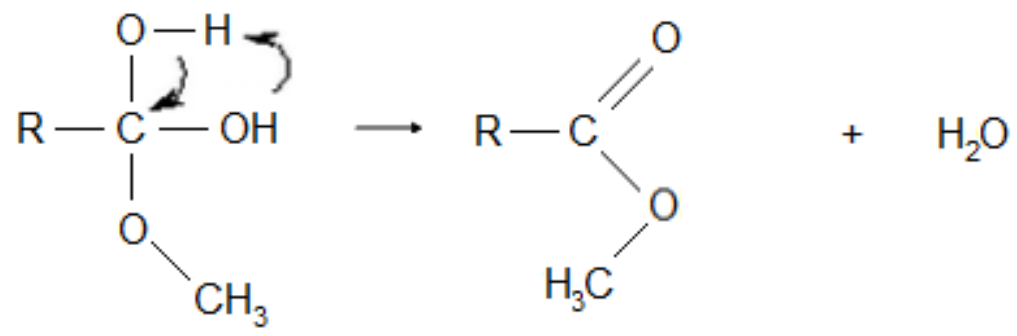

Certas condições, como forte agitação durante a reação, são fatores importantes para a esterificação, uma vez que os radicais precisam interagir para formar os produtos finais (éster e água). É bom lembrar que mais estudos e análises são necessários para compreender todos os aspectos do fotocatalisador e dos reagentes na produção de ésteres.

\section{CONCLUSÕES}

A esterificação por fotocatálise foi apresentada como uma alternativa promissora para a produção de biodiesel. A produção de oleato de metila pela fotocatálise obteve bons resultados na concentração de $3: 1$ de metanol, com uma conversão de $86,0 \%$ utilizando $15 \%$ do catalisador P25. Isto mostra que não é necessário um grande volume de metanol para que uma reação efetiva em éster seja obtida. O uso de um catalisador heterogêneo é um processo mais limpo em comparação com a etapa tradicional, onde se utiliza um catalisador ácido homogêneo. O uso de óleos reais que têm altos níveis de ácidos graxos livres para a esterificação por fotocatálise é uma sugestão para trabalhos futuros.

\section{REFERENCIAS}




\section{9 a 22 de outubro de 2014 \\ Florianópolis/SC}

ALENEZI, R.; SANTOS, R.C.D.; RAYMAHASAY, S.; LEEKE, G.A. Improved biodiesel manufacture at low temperature and short reaction time. Renewable Energy, v. 53, p. 242248, 2013.

ARANDA, D.A.G.; SANTOS, R.T.P.; TAPANES, N.C.O.; RAMOS，A.L.D.; ANTUNES, O.A.C. Acid-catalyzed homogeneous esterification reaction for biodiesel production from palm fatty acids. Catal. Lett., v. 122, p. 20-25, 2008.

CANAKCI, M.; VAN GERPEN, J. Biodiesel production from oils and fats with wigh free fatty acids. Am. Soc. Agr. Eng., v. 44, p. 1429-1436, 2001.

CORRO, G.; TELLEZ, N.; AYALA, E.; MARINEZ-AYALA, A. Two-step biodiesel production from Jatropha curcas crude oil using $\mathrm{SiO}_{2}$.HF solid catalyst for FFA esterification step. Fuel, v. 89, p. 2815-2821, 2010.

CORRO, G.; TELLEZ, N.; JIMENEZ, T.; TAPIA, A.; BANUELOS, F.; VAZQUEZCUCHILLO, O. Biodiesel from waste frying oil. Two step process using acidified $\mathrm{SiO}_{2}$ for esterification step. Catal. Today, v. 166, p. 116-122, 2011.

CORRO, G.; PAL, U.; TELLEZ, N. Biodiesel production from Jatropha curcas crude oil using $\mathrm{ZnO} / \mathrm{SiO}_{2}$ photocatalyst for free fatty acids esterification. Appl. Cat. B: Env., v. 129, p. 3947, 2013.

CUI, W.; XU, C.; ZHANG, S.; FENG, L.; LÜ, S.; QIU, F. Hydrogen evolution by photocatalysis of methanol vapor over Ti-beta. J. Photochem. Photobiol. A: Chem., v. 175, p. 89-93, 2005 .

GOGATE, P.R. E PANDIT, A.B. A review of imperative technologies for wastewater treatment I: oxidation technologies at ambient conditions. Adv. Env. Res., v. 8, p. 501-551, 2004.

HWU, J.R.; HSU, C-Y; JAIN, M.L. Efficient photolytic esterification of carboxylic acids with alcohols in perhalogenated methane. Tetrah. Let., v. 45, p. 5151-5154, 2004.

MARCHETTI, J.M.; ERRAZU, A.F. Comparison of different heterogeneous catalysts and different alcohols for the esterification reaction of oleic acid. Fuel, v.87, p. 3477-3480, 2008.

MEHER, L.C.; VIDYA DAGAR, D.; NAIK, S.N. Technical aspects of biodiesel production by transesterification - a review. Ren. Sust. Energy Rev., v. 10, p. 248-268, 2006.

WU, G., CHEN, T., SU, W., ZHOU, G., ZONG, X., LEI, Z., LI, C. H2 production with ultra-low $\mathrm{CO}$ selectivity via photocatalytic reforming of methanol on $\mathrm{Au} / \mathrm{TiO}_{2}$ catalyst. Int. J. Hydr. Energy., v. 33, p. 1243-1251, 2008.

YAN, S; SALLEY, S.O; SIMON NG, K.Y. Simultaneous transesterification and esterification of unrefined or waste oils over $\mathrm{ZnO}-\mathrm{La}_{2} \mathrm{O}_{3}$ catalysts. Appl. Cat. A: Gen., v. 353, p. 203-212, 